\title{
Relaminarization of a hot air impingement on a flat plate
}

\author{
Mongkol Kaewbumrung ${ }^{1}$ and Chalermpol Plengsa-ard ${ }^{2, *}$ \\ ${ }^{1}$ Faculty of Engineering and Architecture Rajamangala, University of Technology Suvarnabhumi, Ayutthaya, Thailand \\ ${ }^{2}$ Department of mechanical engineering, Kasetsart University, Lat Yao, Chatuchak, Bangkok, Thailand
}

\begin{abstract}
The research mainly focuses on a numerical analysis for heat transfer in the transition flow regimes. The simulation is presented by using ANSYS-FLUENT and Reynolds Averaged Navier Stokes (RANS) technique is employed in order to simulate the complex flow fields. The turbulent jet which impinges on the flat plate with a constant surface heat fluxes is tested. The average Nusselt number predictions are also calculate and compared with existing measurement results. The jet Reynolds number is set to 23,000 which based on jet nozzle diameter, while a jet-toplate spacing of $\mathrm{H} / \mathrm{D}$ is fixed at 2.0. The turbulence models evaluated in the present study are one equation Spalart Allmaras (SA) model, k- $\varepsilon$, shear stress transport (SST) k- $\omega$ and SST with transition model. It can be summarized that the SA, k- $\varepsilon$, and SST k- $\omega$ models fail to calculate the global trend of the instantaneous simulated Nusselt number profiles. Only the simulated results from the SST with transition model provides agree fairly well with experimental results. Moreover, the first highest point of predicted Nusselt number are close to the stagnation point and decrease monotonically in the radial direction within the wall jet region. The second peak of Nusselt number prediction is also observed, and the RANS simulations can capture the relaminarization mechanisms within the boundary layer near walls.
\end{abstract}

\section{Introduction}

For engineering applications, turbulent air jet impingement heat transfer on a wall is commonly employed in a process of cooling and heating materials. In particular, turbulent air jets are ejected from the nozzle exit and high heat transfer rates are initiated when fluid impacts against walls. In the literature review, there are a lot of experimental studied of heat transfer for impinging jets during the last decades. Many reports showed that these heat transfer rates of air jet impingement are dependent on several parameters including jet Reynolds number (Re), the length in radial direction (r/D) from a stagnation point, a nozzle-plate spacing (H/D), and turbulence distribution [1]. A set of experimental data for radial variation of averaged Nusselt number from air jet impingement with Reynolds number of 23,000 were presented by Lytle [2]. One thing can be seen from the previous work, the magnitudes of Nusselt number profiles in radial direction are affected by the nozzle exit to plate spacing (H/D). Moreover, air jet impingement research by Colucci [3] was tested for nozzle to plate spacing $(0.25<\mathrm{H} / \mathrm{D}<6.0)$ and jet Reynolds numbers variation from 10,000 to 50,000 . He concluded that the second peak value of mean Nusselt number in the heat transfer distribution can be observed at low spacing $(\mathrm{H} / \mathrm{D}=0.25)$. A similar report of the second peak with Nusselt number distribution was discovered by Baughn [4]. The second maximum point of the mean Nusselt number distribution occurs when the jets plate spacing is low $(\mathrm{H} / \mathrm{D}<6.0)$. With the flow field point of view, Cooper et al. [5] presented the experimental data of mean velocity distribution and turbulence statistics for air jet impingement from a long pipe. Various numerical results of heat transfer coefficient distributions of impinging jet flows have been investigated in the literature. Wang et al. [6] and Zhou et al. [7] demonstrated the numerical heat transfer analysis of turbulent air jet which impinged on walls. More details of numerical simulations for air jet heat transfer can be found in the literature. However, in summary, all preceding simulated results for Nusselt number prediction do not consistently provide a good agreement with measurement data. Only a few reports were focused on a computational modeling of transitional jet impingement. The objective of this research is to propose appropriate turbulence models for transition flows with RANS simulations. Clearly, the novel transition SST models can predict more accurate average Nusselt number results and to capture the second peak of Nusselt number in transition wall flows. These predicted results may help to understand the detailed local information of air jet impingement near walls.

\section{Description of the problem}

The jet impingements on heated wall are investigated and air is selected to be a working fluid. A long pipe with a thin wall is considered to be a nozzle with

\footnotetext{
* Corresponding author: fengcpp@ku.ac.th
} 
diameter $\mathrm{D}=26.5 \mathrm{~mm}$, and the flow patterns of air jet from the nozzle exit are in the vertical direction. The normal distance from nozzle exit to a wall surface is fixed at $H / D=2$ and a dimension of the bottom circle high temperature surface is about $16 \mathrm{Dx} 16 \mathrm{D}$ which is far enough from the outlet pressure effect. The schematic of computational domain for this problem are demonstrated in Figure 1. Also, the reference axis $\mathrm{x}$ and $\mathrm{r}$ axis are included. The internal averaged air velocity is equal to $15.54 \mathrm{~m} / \mathrm{s}$, while the air density is set to be $1 \mathrm{~kg} / \mathrm{m}^{3}$. Incompressible flow assumptions are valid for this study due to low Mach number condition.

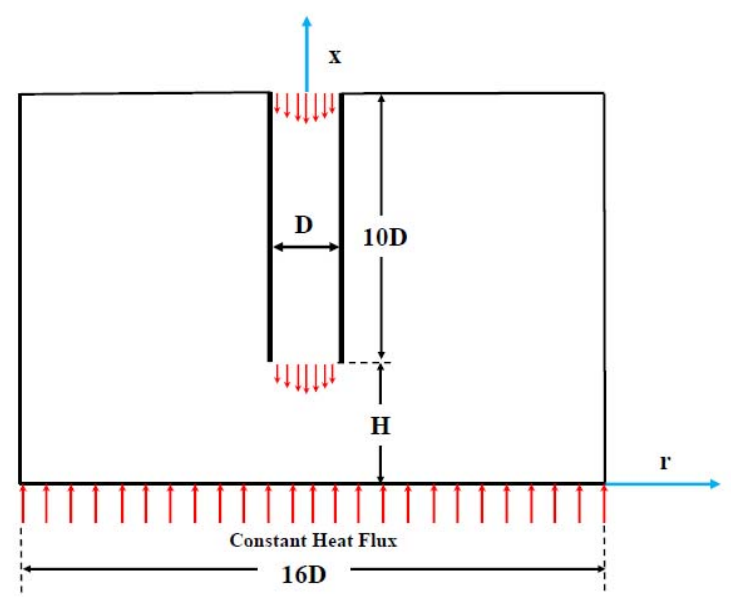

Fig. 1. A computational domain of jet impingement

\section{Mathematical formulations}

The impinging jet flow phenomena reveals that both laminar and turbulent flow happens concurrently in different regions. The CFD solution from the momentum and continuity equations with appropriate CFD grids are enough to resolve the laminar flow phenomenon near walls. However, for transitional or turbulent wall bounded flows, a small scale flow structure is modeled and a turbulence model is used. In particular, turbulent motions are unsteady and strongly affect the global flow structures of velocity fields, thus the accurate prediction of turbulent flows is desired. To simulate turbulent flow fields, the numerical flow solutions are calculated from solving the classical conservation laws such as mass, momentum and energy governing equations, together with the turbulence model selection. In addition, the present investigation uses a novel transition SST model which employs new empirical correlations and were developed by Langtry and Menter [8]. In particular, The gamma or intermittency factor $(\gamma)$ and the transition momentum thickness Reynolds number $\left(\operatorname{Re}_{\theta}\right)$ are directly solved. The intermittency factor results is a production term for the turbulent kinetic energy transport equation in SST turbulence model. More details for transition SST model can be found in the literature.

\section{Solution methodology}

To match with experimental conditions, air jet impingement which is a normal direction to a flat wall with Reynolds number $=23,000$ is tested. The internal velocity profiles are assumed a fully developed with no slip conditions. Also, the pipe wall boundary conditions are adiabatic. The thermal boundary conditions on the flat surfaces are constant heat fluxes of $300 \mathrm{~W} / \mathrm{m}^{2}$, while the present studies use the ambient outlet pressure boundary conditions. The simulation is presented by using CFD commercial code namely ANSYS-FLUENT. To simulate the flow field, time average techniques (RANS) simulations with transition SST models are employed. The coupled algorithm for pressure-velocity and Green-Gauss cell based scheme for gradients are also incorporated. The first grid point $\mathrm{y}$ plus $(\mathrm{y}+)$ from the wall is $0.04(<1)$ which is adequate to resolve flow fields near walls. The total computational fluid cells are 5.4 million cells and the CPU time is about 24 hours with 16 cores. The average Nusselt number prediction is defined by the following equation:

$$
N u=\frac{q_{W} D}{\left(T-T_{r e f}\right) k}
$$

The film temperature which is defined as the below equation is used to obtain the physical properties.

$$
T_{f}=\frac{1}{2}\left(T+T_{r e f}\right)
$$

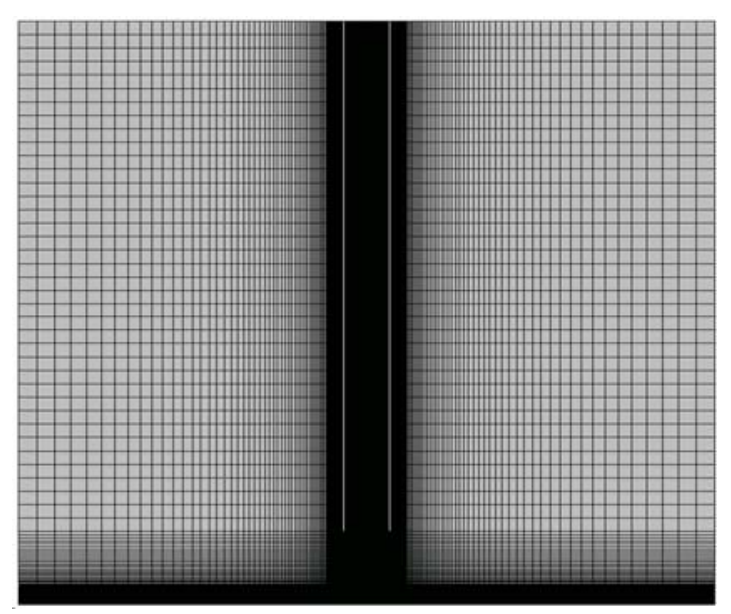

Fig. 2. The front view of CFD mesh 


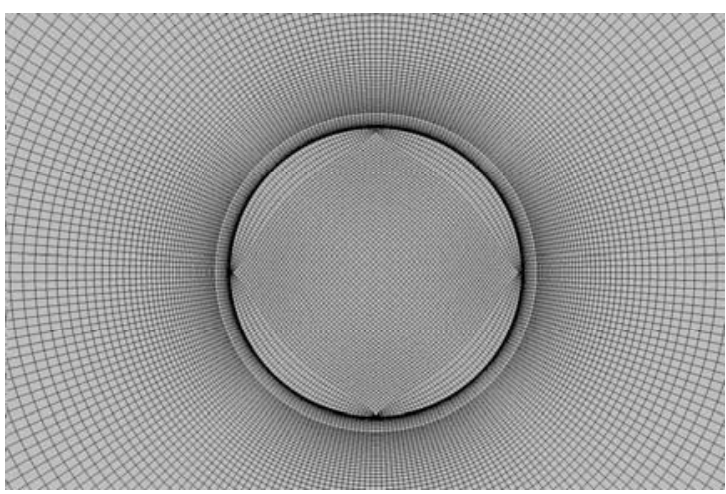

Fig. 3. The nozzle inlet of CFD mesh

\section{Results and discussion}

This section consists of three parts. In the first part, the predicted results of mean Nusselt number on a hot flat plate are shown. The available experimental data from Baughn [4] are also plotted for comparison. In the second part, normalize mean jet velocity versus the axial vertical distance are demonstrated. Finally, the contour plots of Nusselt number and velocity profiles near walls in transition wall zones are presented.

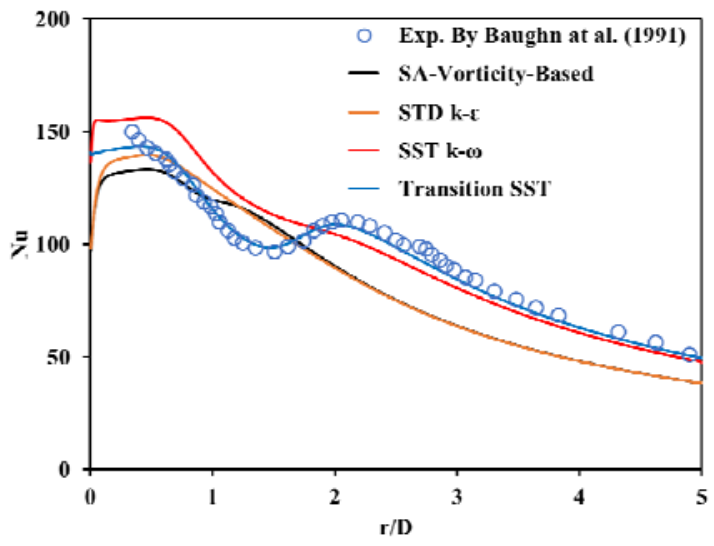

Fig. 4. The predicted and measured mean Nusselt number versus radial distance with different turbulence models comparisons.

Figure 4 illustrates the predicted and measured mean Nusselt number with various turbulence models for comparison. Clearly, a stagnation zone $(\mathrm{r} / \mathrm{D}<0.1)$ is formed directly under the jet where it impacts the surface. This stationary flow is accelerated, and after being laminar initially, then transitions and becomes turbulent. The initial acceleration and associated convection cause a high convection heat transfer zone, but, because the flow is laminar, no mixing occurs and the heat transfer rate drops at increasing radii. After transition, however, the turbulent flow with its associated mixing enhances heat transfer which leads to another local maximum zone of heat transfer. It is clear that simulated results from transition SST model are good agreement with measurement data. The near wall treatment from SST transition model can capture the second peak of Nusselt number. The intermittency factor play an important role in the turbulent kinetic energy transport equation in SST models. As predicted by SST transition model shown in Figures 5-7, the simulated and measured normalized mean axial and radial velocity components are demonstrated at radial position $\mathrm{r} / \mathrm{D}=$ $0.5,1.0$ and 1.5. The data from Cooper et. al. [5] are included and compared. The simulated velocity profiles are fairly agreement when compared those of experimental results. At $\mathrm{r} / \mathrm{D}=0.5$ near a stagnation zone, the normalized velocity profiles are almost uniform. However, the velocity fluctuations (more mixing) can be seen in Figures 6 and 7. The highest values of normalized mean velocity arises at $r / D=1.0$ which are consistent with the location of the highest values of Nusselt number.

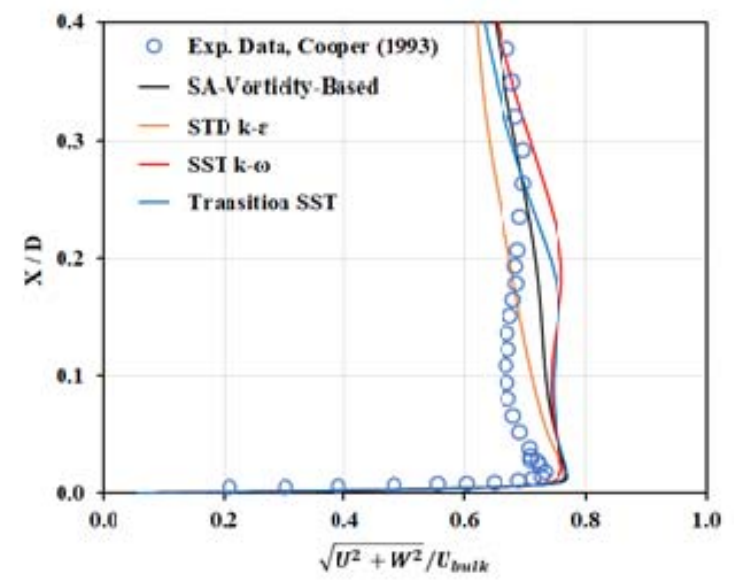

Fig. 5. The simulated and measured normalized mean jet velocity at $\mathrm{r} / \mathrm{D}=0.5$ as predicted by $\mathrm{SST}$ transition model.

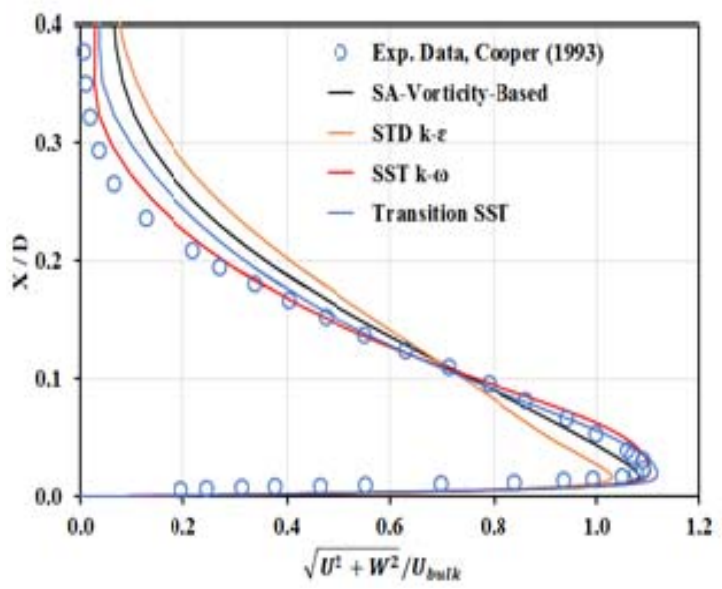

Fig. 6. The simulated and measured normalized mean jet velocity at $\mathrm{r} / \mathrm{D}=1.0$ as predicted by SST transition model. 


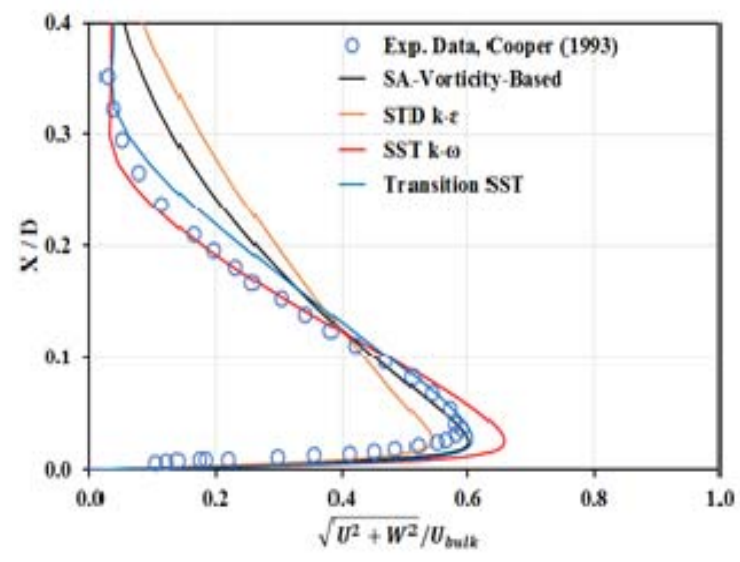

Fig. 7. The simulated and measured normalized mean jet velocity at $\mathrm{r} / \mathrm{D}=1.5$ as predicted by SST transition model.

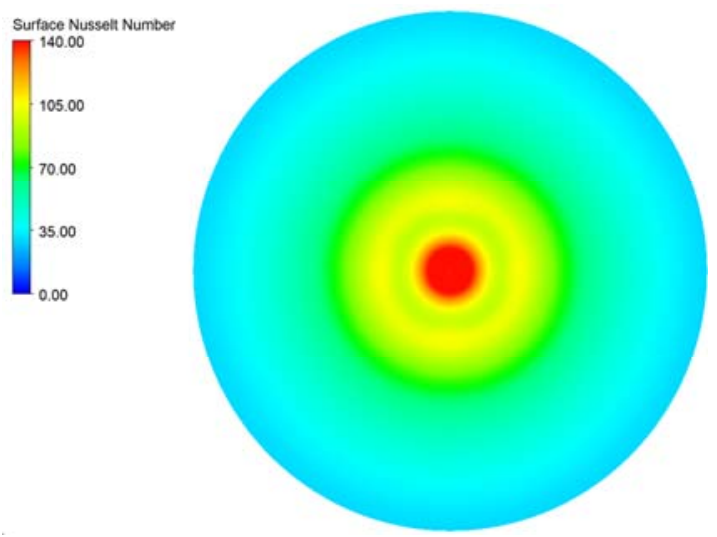

Fig. 8. Contour of surface average Nusselt number as predicted by SST transition model.

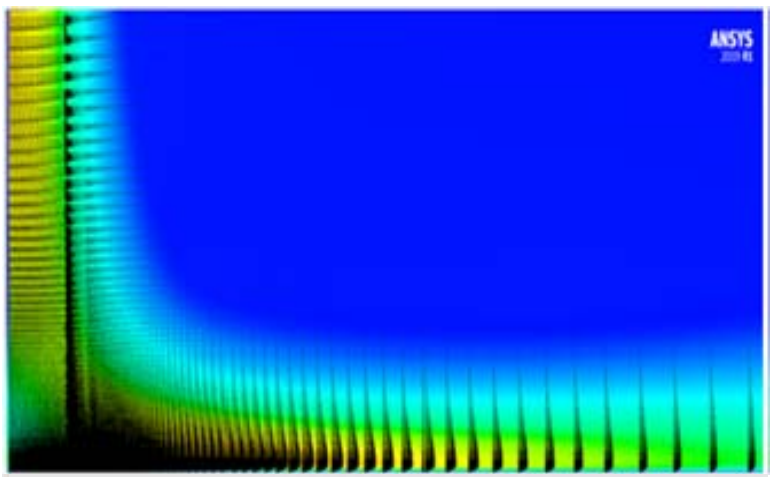

Fig. 9. The redistribution of velocity profiles near walls as predicted by SST transition model.

Figure 8 illuminates the contour plots of mean Nusselt number on the flat plate using SST transition model. The mean Nusselt number prediction values are started from 0.1 to 140 , while the highest value of non-dimension heat transfer rate is close to the stagnation point. The contour of mean Nusselt number is obviously nonuniform and the second peak of Nusselt number is observed from a yellow color ring. For SST transition models, the redistribution of velocity profiles of laminar to transition and turbulent flows are demonstrated in Figure 9. The no-slip velocity boundary condition also is observed.

\section{Conclusions}

The research mainly focuses on a numerical study for turbulent flow and heat transfers in the transition flow regimes of air jet impingement. The present study use the commercial code ANSYS FLUENT was used to simulate jet impingement on a flat plate and RANS techniques were employed to simulate the flow field. For model validation, the simulated results were validated with available experimental data from the literature. The constant heat flux thermal boundary condition was chosen to be a test case. The jet Reynolds number is equal to 23,000 and a fixed jet-to-plate spacing of $\mathrm{H} / \mathrm{D}=$ 2.0. From the investigation, we summarize as follow:

1) The one equation Spalart Allmaras (SA), k- $\varepsilon$, and SST $\mathrm{k}-\omega$ models are unsuccessful to calculate the global trend of the mean simulated Nusselt number profiles.

2) Only the simulated results from the SST with transition model provides a good agreement with measurement results.

3) The first maximum point of simulated Nusselt number values is near the stagnation point and the magnitudes of Nusselt number prediction decrease monotonically in the radial direction within the wall jet region.

4) The second peak of Nusselt number prediction from the transition SST model is observed.

This study is based upon work supported by mechanical engineering department faculty of engineering Kasetsart University. Also, the authors are grateful for the intellectual support from Dr. Chalermpol Plengsa-ard.

\section{References}

1. K. Jambunathan, E. Lai, M.A. Moss, and B.L. Button, Int. J. Heat Fluid Flow, 13, 106-115 (1992)

2. D. Lytle and B.W. Webb, In Experimental Heat Transfer, Fluid Mechanics and Thermodynamics (ed. J. F. Keffer, R. K. Shah \& E. N. Ganic), Elsevier. (1991)

3. D. Colucci, and R. Viskanta, Expl Thermal Fluid Sci., 13, 71-80 (1996)

4. J. Baughn and S. Shimizu, ASME J. Heat transfer, 111 , 1096-1098 (1989)

5. D. Cooper, D.C. Jackson, B. Launder, and G.X. Liao, Int. J. Heat and Mass Transfer, 36, 2675-2684 (1993)

6. B. Wang, D. Lin, Q. Xie, Z. Wang, and G. Wang, Applied Thermal Engineering, 100, 902-910 (2016)

7. T. Zhou, D. Xu, J. Chen, C. Cao, and T. Ye, Applied Thermal Engineering, 100 , 55-61 (2016)

8. Langtry R. B., and Menter F. R., 43rd AIAA Aerospace Sciences Meeting and Exhibit 2005, Reno, Nevada, 522.1-14 (2005) 